\title{
Eosinophil-mediated lung inflammation associated with elevated natural killer T cell response in COVID-19 patients
}

Dong-Min Kim 1,*, Jun-Won Seo ${ }^{1,}$, Yuri Kim², Uni Park ${ }^{2}$, Na-Young Ha ${ }^{2}$, Hyoree Park ${ }^{2}$, Na Ra Yun ${ }^{1}$, Da Young Kim', Sung Ho Yoon ${ }^{1}$, Yong Sub Na', Do Sik Moon', Sung-Chul Lim³ ${ }^{3}$ Choon-Mee Kim ${ }^{4}$, Yeon-Sook Kim ${ }^{5}$, and Nam-Hyuk Cho ${ }^{2}$

${ }^{1}$ Department of Internal Medicine, Chosun University College of Medicine, Gwangju; ${ }^{2}$ Department of Microbiology and Immunology, Seoul National University College of Medicine, Seoul; Departments of ${ }^{3}$ Pathology and ${ }^{4}$ Premedical Science, Chosun University College of Medicine, Gwangju; ${ }^{5}$ Department of Internal Medicine, Chungnam National University College of Medicine, Daejeon, Korea

\section{Eosinophil-mediated lung inflammation associated with elevated natural killer T cell response in COVID-19 patients}

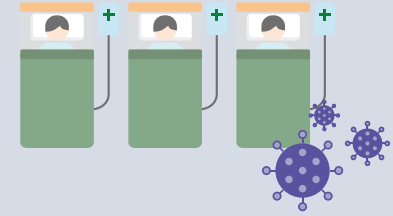

3 COVID-19 pneumonia patients

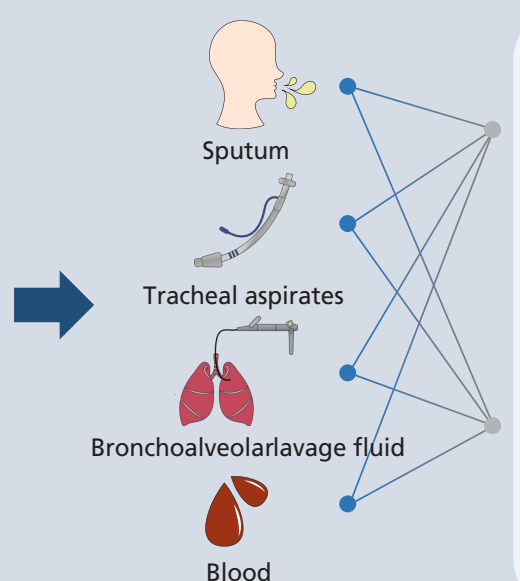

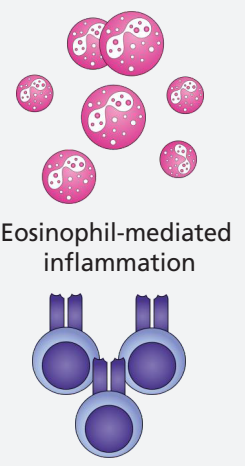

NKT cell elevation

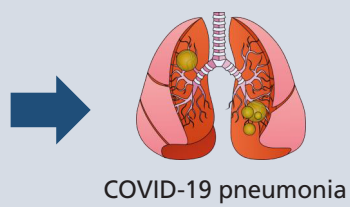

COVID-19 pneumonia

Lungs of COVID-19 patients can exhibit eosinophil-mediated inflammation, together with an elevated NKT cell response.

Received : February 16, 2021

Revised : July 7, 2021

Accepted: July 16, 2021
Correspondence to Nam-Hyuk Cho, Ph.D.

Department of Microbiology and Immunology, Seoul National University College of Medicine, 103 Daehak-ro, Jongno-gu, Seoul 03080, Korea

Tel: +82-2-740-8392, Fax: +82-2-743-0881, E-mail: chonh@snu.ac.kr

https://orcid.org/0000-0003-3673-6397

*These authors contributed equally to this work. 
Background/Aims: Coronavirus disease 2019 (COVID-19) is associated with acute respiratory syndrome. The mechanisms underlying the different degrees of pneumonia severity in patients with COVID-19 remain elusive. This study provides evidence that COVID-19 is associated with eosinophil-mediated inflammation.

Methods: We performed a retrospective case series of three patients with laboratory and radiologically confirmed COVID-19 pneumonia admitted to Chosun University Hospital. Demographic and clinical data on inflammatory cell lung infiltration and cytokine levels in patients with COVID-19 were collected.

Results: Cytological analysis of sputum, tracheal aspirates, and bronchoalveolar lavage fluid (BALF) samples from all three patients revealed massive infiltration of polymorphonuclear cells (PMNs), such as eosinophils and neutrophils. All sputum and BALF specimens contained high levels of eosinophil cationic proteins. The infiltration of PMNs into the lungs, together with elevated levels of natural killer T (NKT) cells in BALF and peripheral blood samples from patients with severe pneumonia in the acute phase was confirmed by flow cytometry.

Conclusions: These results suggest that the lungs of COVID-19 patients can exhibit eosinophil-mediated inflammation, together with an elevated NKT cell response, which is associated with COVID-19 pneumonia.

Keywords: COVID-19; SARS-CoV-2; Eosinophil; Natural killer T-cells; Bronchoalveolar lavage

\section{INTRODUCTION}

In December 2019, a novel coronavirus was identified as the cause of pneumonia in a cluster of cases in Wuhan, China [1]. This virus, which causes coronavirus disease 2019 (COVID-19), was identified as severe acute respiratory syndrome coronavirus-2 (SARS-CoV-2) [2]. Today, approximately $80 \%$ of confirmed COVID-19 cases are asymptomatic or lead to mild symptoms in infected individuals. However, in the remaining $20 \%$ of cases severe pneumonia often developed, with patients requiring supplemental oxygen therapy $[3,4]$. The mechanisms underlying the different degrees of pneumonia severity observed in patients with COVID-19 remain elusive.

To characterize the cause of pneumonia in patients with COVID-19, we analyzed the immunological features of respiratory and blood specimens collected from three patients with confirmed infection by SARS-CoV-2 but varying degrees of clinical symptoms: one patient with mild symptoms without pneumonia and two patients with severe pneumonia. We analyzed plasma, leukocytes in blood, bronchoalveolar lavage fluids (BALFs), sputa, and tracheal aspirates to characterize the immunological responses to viral infection. We propose the possibility of one of several immune mechanisms of COVID-19 by reporting eosinophil-mediated lung inflammation associated with elevated natural killer T (NKT) cell response in patients with COVID-19.

\section{METHODS}

\section{Ethnical statement}

The present study was approved by the Institutional Review Boards of Chosun University Hospital (IRB no.: 202002-011) and Seoul National University Hospital (IRB no.: C-1509-103-705). This study was conducted with informed consent from the patients or their legal guardians.

\section{Patient characteristics}

General information on the clinical courses and baseline characteristics of the three patients included in this study are summarized in Table 1 and Fig. 1A. A 79-year-old man (P1) developed fever and headache after contact with his daughter, who had confirmed COVID-19. A reverse transcriptase-polymerase chain reaction (RT-PCR) test for SARSCoV-2 genes was performed on the first day after symptom onset, which confirmed that P1 was COVID-19 positive. On day 5 after symptom onset, P1 was admitted to a local hospital and was administered lopinavir/ritonavir. On day 9 , the patient developed acute respiratory distress syndrome (ARDS); consequently, mechanical ventilation was initiated. On day 12, bronchoscopy with BAL was performed. Corticosteroids were administered on day 17 based on the BALF results. A follow-up chest $X$-ray was performed on day 2 after corticosteroid administration (Fig. 1B), which showed significant improvement in pneumonic infiltration and a decrease in $\mathrm{O}_{2}$ demand (fraction of inspired oxygen $\left[\mathrm{FiO}_{2}\right]$, 
from 0.8 to 0.4. A 79-year-old woman (P2) was confirmed to be COVID-19 positive and asymptomatic. Two days later, the patient developed dyspnea with gradual aggravation and showed an increase in $\mathrm{O}_{2}$ demand. She had fever $\left(38.3^{\circ} \mathrm{C}\right)$ and showed pneumonic infiltration in both lung fields (Fig. 1B). Mechanical ventilation was initiated at an $\mathrm{O}_{2}$ saturation of $85 \%$ due to persistent hypoxemia despite the application of high-flow nasal cannula therapy. Lopinavir/ ritonavir was administered from the first day of symptom onset, but there was no change in the severity of pneumonia. Therefore, bronchoscopy with bronchoalveolar lavage was performed on day 13. On day 14, corticosteroids were administered based on BALF results. Follow-up chest radiography was performed on day 16 (Fig. 1B), which indicated signs of pneumonia resolution. Although a sharp increase in blood sugar levels was observed following steroid administration, the $\mathrm{O}_{2}$ demand decreased $\left(\mathrm{FiO}_{2}\right.$ from 0.7 to 0.4$)$. A 36-year-old man (P3) with no underlying disease conditions and symptoms visited the COVID-19 screening clinic because he had returned from a COVID-19 endemic country. RT-PCR analysis confirmed that the patient was COVID-19 positive. At the time of hospitalization, pneumonia was not detected on chest radiography (Fig. 1B) or high-resolution computed tomography. The patient complained of a sore throat the day after admission and developed a fever (approximately $38^{\circ} \mathrm{C}$ ). Hydroxychloroquine treatment was initiated on day 4 when P3 developed a productive cough with fever.

Chest radiographic scores were calculated using X-rays. The scores were calculated by dividing each lung into the upper, middle, and lower zones. Each zone was assigned a score from 0 to 4 points based on the degree of infiltration. The scores for the six zones were then tallied, yielding a total score ranging from 0 to 24 [5]. All remaining experimental methods are listed in the Supplementary methods.

\section{RESULTS}

The clinical courses and baseline characteristics of the three patients are summarized in Table 1 and Fig. 1A. P1 and P2 had severe pneumonia and required mechanical ventilation (Fig. 1B). P3 had only mild symptoms, without pneumonia. None of the three patients had a history of eosinophilia due to conditions, such as asthma, or allergy.

Initially, we analyzed BALFs and sputa to identify immune cells that had infiltrated the infected lungs. The cytological

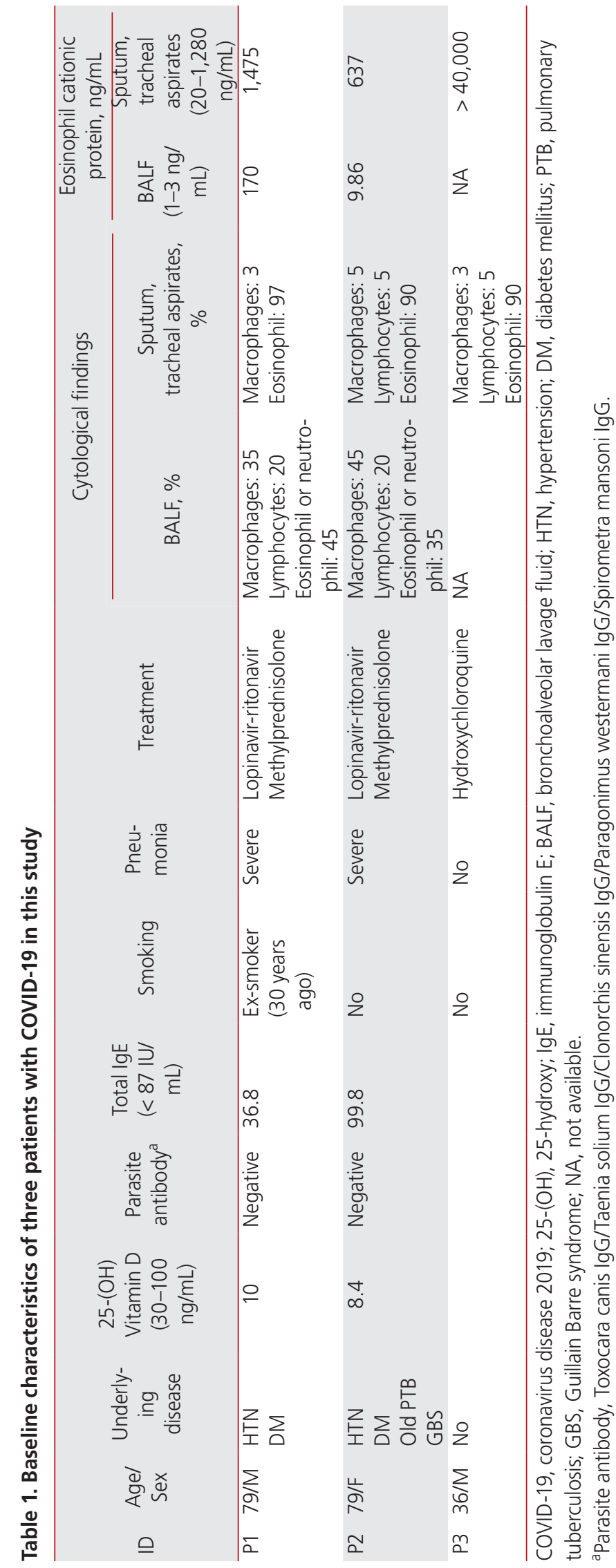




\section{KJIMT}

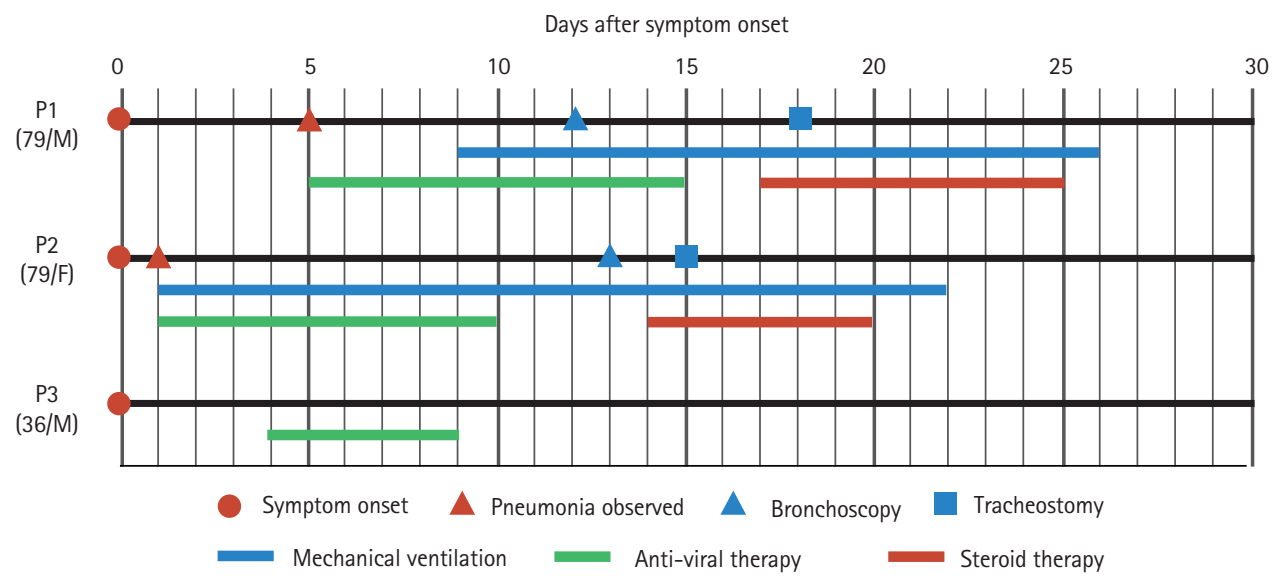

P1
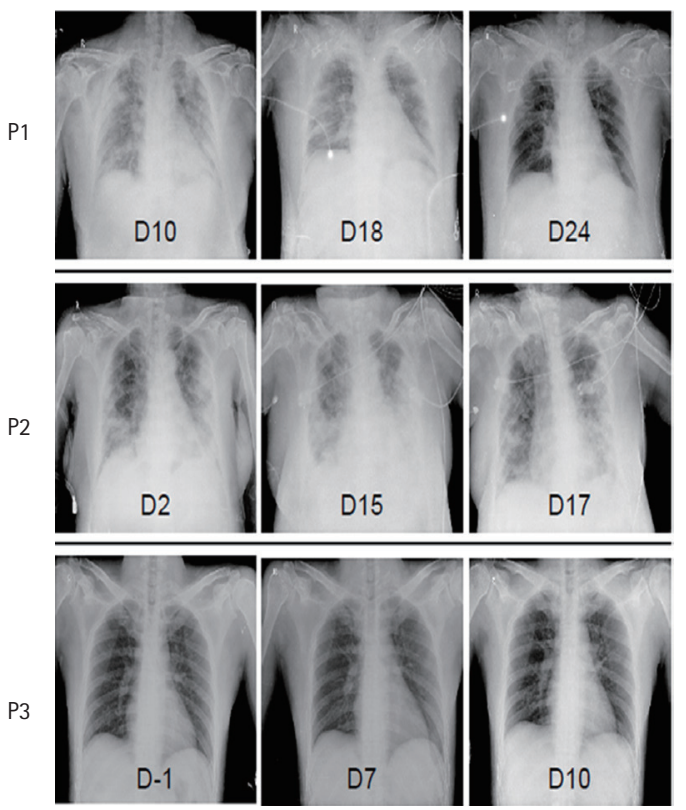

B
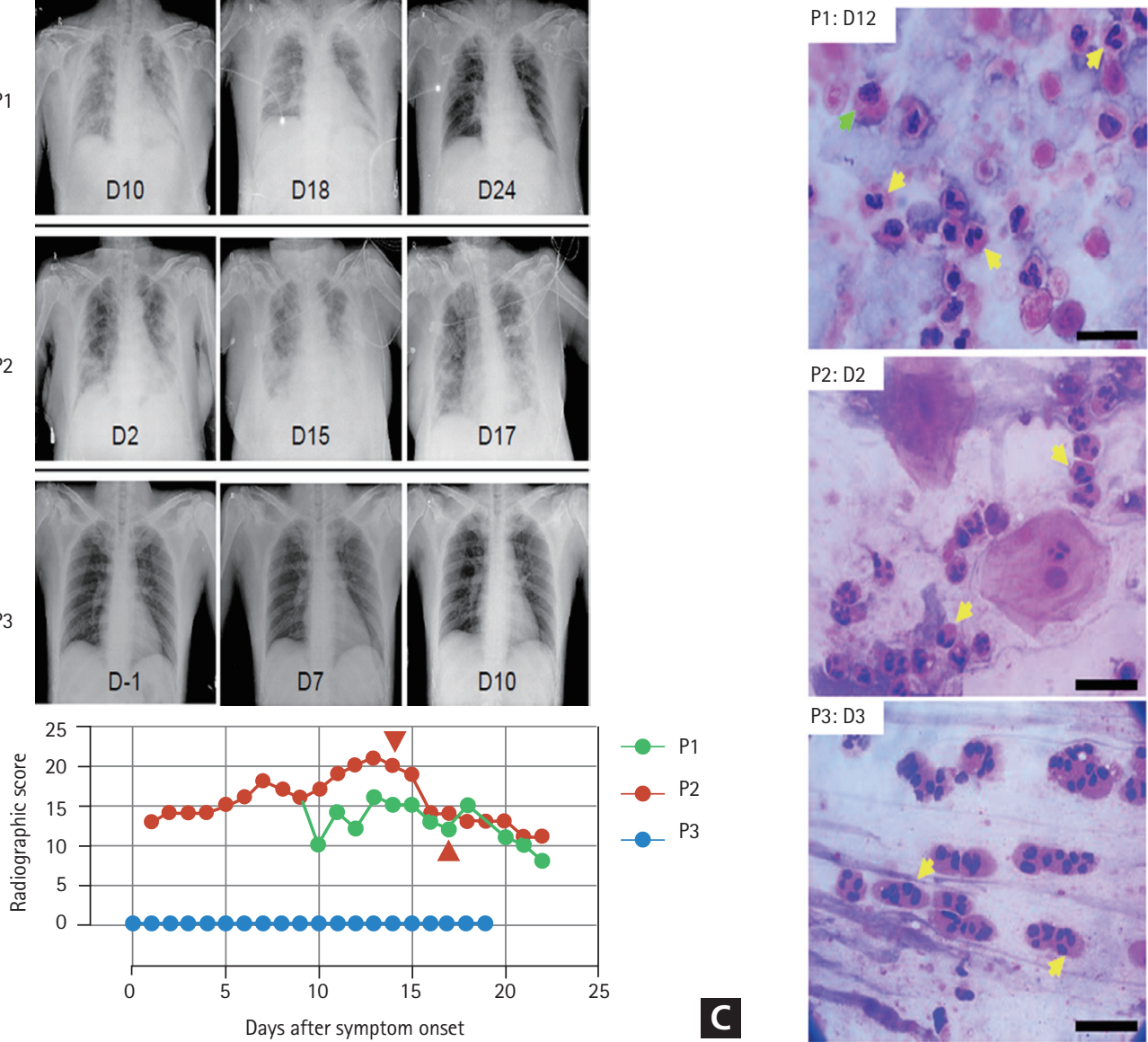

Figure 1. Clinical courses of three coronavirus disease 2019 (COVID-19) patients enrolled in the present study. (A) Schematic diagram of the clinical course of the patients and the administered treatments. (B) Representative chest images (upper images) and radiographic scores (lower graph) of patients with COVID-19. Images were acquired on the indicated days after symptom onset. Brown arrowheads indicate the commencement of steroid therapy. (C) Representative images of the cytological analysis of bronchoalveolar lavage fluid (P1, upper) and sputum (P2, middle; P3, lower) specimens by H\&E staining. Images were acquired on the indicated days after symptom onset. Arrows indicate polymorphonuclear cells (yellow) and macrophages (green). Scale bar: $20 \mu \mathrm{m}$. 
analyses of BALF and sputum were performed using hematoxylin and eosin (H\&E) staining, which showed that polymorphonuclear cells (PMNs), especially eosinophils or neutrophils, were the major inflammatory cells, along with a few macrophages and lymphocytes (Table 1 and Fig. 1C). BALF analysis of the two patients that developed severe pneumonia (P1 and P2) showed that lymphocytes accounted for $20 \%$ and PMNs accounted for more than $35 \%$ of the inflammatory cells. Eosinophils accounted for more than $25 \%$ of the PMNs. Eosinophils constituted less than $1 \%$ of BALF obtained from healthy individuals. Acute eosinophilic pneumonia is diagnosed when eosinophils constitute more than $25 \%$ of the BAL differential count in the absence of other causes of BALF eosinophilia, such as asthma or atopic disease, and drugs [6]. Our study revealed that COVID-19 could cause eosinophil-mediated inflammation in the lungs, similar to acute eosinophilic pneumonia.

Cytological analysis of sputum and tracheal aspirates collected from the three patients revealed that more than $90 \%$ of the cells were eosinophils (Table 1 and Fig. 1C). Notably, a sputum sample was collected from P3 (who did not develop pneumonia) on day 3, after symptom onset, also showed high levels of eosinophils. Additionally, a sputum sample obtained from $\mathrm{P} 2$, who rapidly developed severe pneumonia on day 2 , also contained a large number of PMNs (Table 1 and Fig. 1C). Therefore, infiltration of PMNs into infected lungs might be rapid and universal in patients with COVID-19, regardless of the severity of pneumonia.

Based on the cytological analysis, we suspected that among the other PMNs, eosinophils infiltrated the lungs. Therefore, we measured the levels of eosinophil cationic proteins (ECPS) in the respiratory specimens. The sputum and BALF specimens from the three patients contained high levels of ECP (Table 1), which indicated the infiltration of eosinophils into the inflamed lungs during the acute phase of COVID-19. To further characterize the PMNs infiltrating the pneumonic lungs, BALF was collected from P1 on day 12 (acute phase) and 19 days (convalescent phase) after symptom onset and subjected to cytometric analysis. Peripheral blood collected from P2 on day 12 (acute phase) and P1 on day 19 (convalescent phase) after symptom onset were simultaneously used for flow cytometric analysis of leukocytes. We identified the relative proportions and kinetic changes in $\mathrm{CD}_{14}{ }^{+}$monocytes/macrophages, $\mathrm{CD}^{+} \mathrm{T}$ cells, CD20+ B cells, and side scatter (SSC) ${ }^{\text {high }} /$ CD24 $4^{+}$PMNs, including neutrophils and eosinophils, in the blood leuko- cytes and BALF (Supplementary Fig. 1). The BALF from P2 showed a marked decrease in the number of $C D 45^{+}$leukocytes during the convalescent phase $\left(\sim 2.5 \times 10^{3} / \mathrm{mL}\right)$ of the disease, compared to that in the acute phase $\left(\sim 1.3 \times 10^{5}\right)$ $\mathrm{mL}$ ). Similar to the cytological analysis of BALF, the relative proportion of PMNs in the CD45+ leukocyte populations (after excluding macrophages, $T$ cells, and B cells) increased in the acute phase $(30.5 \%)$ and decreased to $10.6 \%$ in the convalescent phase. Although the PMNs in BALF collected during the acute phase were primarily composed of $\mathrm{CD}_{16}{ }^{+} /$ CD24+ neutrophils (26.8\% in CD45+ leukocytes), approximately 10\% (3.0\% in CD45+ leukocytes) of the PMNs were likely to be $\mathrm{CD} 24^{+}$eosinophils with reduced expression of CD16 on the surface (Supplementary Fig. 1) [6]. Monocytes in the peripheral blood increased during the acute phase but decreased during the convalescent phase. However, of the total leukocytes in the BALF, monocyte counts decreased during the acute phase and increased during the convalescent phase. Although very few $B$ cells were detected in the BALF during the acute phase, their numbers increased in the convalescent phase. Interestingly, most $T$ cells in the acute phase BALFs showed intermediate levels of surface CD3 molecules compared to those of blood T cells, indicating the presence of other types of $\mathrm{CD}^{+}$lymphocytes. We assessed the surface expression of other cellular markers, such as CD24, CD16, and human leukocyte antigen DM (HLA-DM), and found that the majority of the $C D 3^{+} T$ cells in the acute phase of BALF were CD16 $/$ CD24 $/ / \mathrm{HLA}^{-} \mathrm{DM}^{-}$ cells, suggesting a predominantly NKT cell population (the last row of Supplementary Figs. 1 and 2A) [7,8]. An increase in the number of NKT cells expressing both CD3 and CD56 in the patient's blood during the acute phase was further confirmed by flow cytometric analysis (Fig. 2A, 2B, and Supplementary Fig. 2B) of peripheral blood mononuclear cells (PBMCs). The relative levels of $\mathrm{CD}^{+} / \mathrm{CD} 6^{+} \mathrm{NKT}$ cells were 6.4-and 2.5 times higher in the patients' blood lymphocytes during the acute phase $(5.8 \% \pm 1.3 \%$, mean \pm standard deviation) than in healthy controls $(0.9 \% \pm 0.8 \%)$ and patients in the convalescent phase $(2.3 \% \pm 1.3 \%)$, respectively. Additionally, the $\mathrm{CD}^{+} / \mathrm{CD} 1 \mathrm{~d}$-tetramer ${ }^{+} \mathrm{NKT}$ (invariant NKT [iNKT]) [9] cells at acute phase $(1.3 \pm 0.4)$ were significantly elevated by 4.3 and 1.6 times compared to those of healthy controls $(0.3 \pm 0.1)$ and convalescent phase $(0.8 \pm 0.1)$, respectively (Fig. 2A, 2B, and Supplementary Fig. 2B). However, the levels of $C D 3^{-} / C^{2} 56^{+}$NK cells in the peripheral blood changed depending on the individual cases (Supplementary 


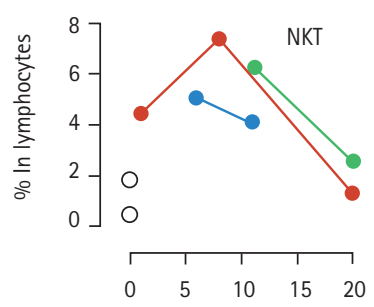

A

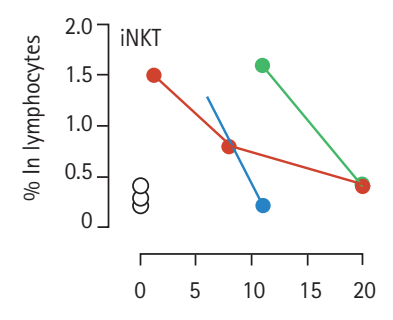

Days after symptom onset

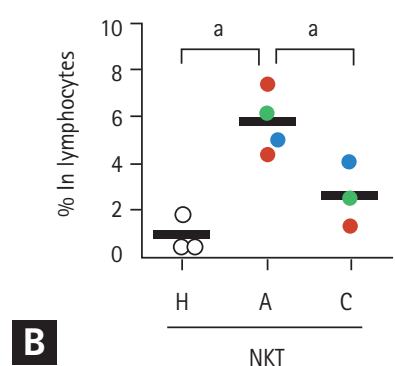

B

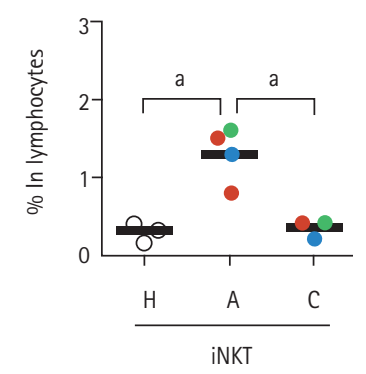

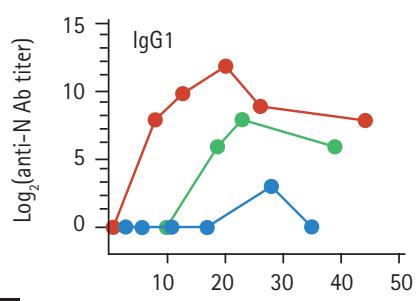

C

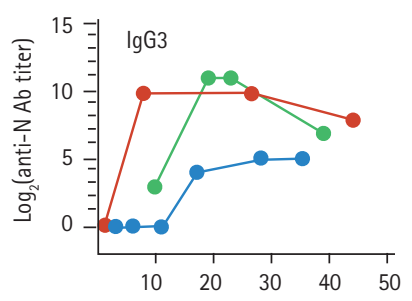

Days after symptom onset

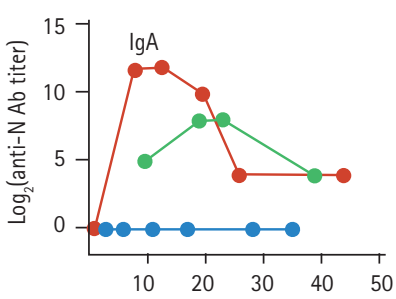

Days after symptom onset

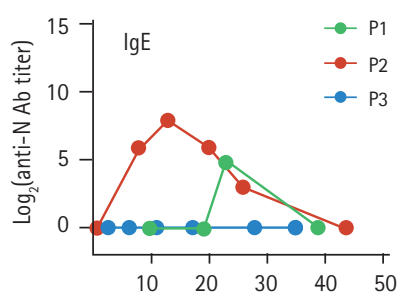

Days after symptom onset

Figure 2. Kinetic changes in natural killer T (NKT) cell frequencies in peripheral lymphocytes and severe acute respiratory syndrome coronavirus-2 (SARS-CoV-2) N-specific antibodies in the plasma from patients with coronavirus disease 2019 (COVID-19). (A) Kinetic changes in the relative frequencies of $\mathrm{CD}^{+} / \mathrm{CD}_{56}{ }^{-} \mathrm{NKT}$ and $\mathrm{CD}^{+} / \mathrm{CD} 1 \mathrm{~d}$-tetramer ${ }^{+}$invariant NKT (iNKT) cells among the lymphocytes from the indicated patients. The relative frequencies of each immune cell type from three healthy volunteers were used as controls (open circles). (B) The relative frequencies of $\mathrm{CD}^{+} / \mathrm{CD}_{5} 6^{+} \mathrm{NKT}$ and $\mathrm{CD}^{+} / \mathrm{CD} 1 \mathrm{~d}$-tetramer ${ }^{+}$iNKT cells among the lymphocytes from the indicated patients were rearranged based on the collection periods; i.e., acute phase $(A)$ and convalescent phase $(C)$, and compared using one-way analysis of variance (ANOVA) followed by Newman-Keuls $t$ test. The relative frequencies of each immune cell type from three healthy volunteers were considered as controls ( $\mathrm{H}$, open circles). Black bars indicate the mean value, and $p$ values were obtained using ANOVA followed by Newman-Keuls $t$ test. (C) Kinetic changes in specific antibodies against the viral N protein are presented. The specific isotypes of the antigen-specific antibodies have been indicated. Ig, immunoglobulin. ${ }^{a} p<0.01$.

Fig. 2B and 2C). Quantitative changes in the NKT cell population were consistently observed in the three patients, suggesting that upregulation of the NKT cell population might be a common phenotype initiated upon SARS-CoV-2 infection regardless of disease severity. Furthermore, compared to the other lymphocytes, the relative frequency of NKT cells among lymphocytes decreased during the convalescent phase, which suggests that the kinetic changes in NKT cells might be correlated with disease progression.

\section{DISCUSSION}

Significant elevation and infiltration of NKT cells and PMNs, including neutrophils and eosinophils, into the lungs of patients with COVID-19 during the acute phase of infection indicated that these innate immune cells might be the initial mediators of pulmonary inflammation. This type of immunopathological response appears to commonly occur primarily in patients with symptomatic COVID-19 regardless of disease severity, as a similar degree of eosinophilic infiltration was observed in P3, who did not have pneumonia. Several studies have recently been published on the composition of inflammatory cells in the BALF of individuals with severe COVID-19. Dentone et al. [10] reported that neutrophils and macrophages were the major cell types in the BALF of patients with severe COVID-19, whereas lymphocytes were a minority. Furthermore, they identified a correlation between the percentage of macrophages in the BALF and patient mortality [10]. Liao et al. [11] reported that the BALF of patients with severe COVID-19 had higher levels of macrophages and neutrophils and lower proportions of $T$ and NK lymphocytes than those of patients with moderate disease severity. Pandolfi et al. [12] performed a BALF analysis of 
patients with severe COVID-19 and observed high levels of neutrophils with very few lymphocytes and macrophages. Although these studies did not investigate the role of eosinophils, our current data indicate the potential involvement of eosinophil-mediated inflammation in COVID-19 patients.

Eosinophils are known to play a role in the immune response against various viral infections. Fraenkel et al. [13] reported that the common cold, caused by rhinovirus, is associated with eosinophilic infiltration of the bronchial mucosa. Harrison et al. [14] also showed that respiratory syncytial virus (RSV) infection induced ECP production in the lower airway and suggested that eosinophil degranulation plays a role in the pathogenesis of respiratory infection by RSV. In other words, given the various roles that eosinophils play in host immune responses, it may be suggested that viral infections cause an increase in eosinophil counts at the site of infection. Jeon et al. [15] reported a case of acute eosinophilic pneumonia associated with influenza A (H1N1) infection in 2009. Subsequently, LeMessurier et al. [16] showed that eosinophils contribute to antiviral host defense mechanisms against respiratory infections caused by the 2009 influenza A (H1N1) infection.

Cavalli et al. [17] showed that the relative proportions of infiltrating immune cells, such as NKT cells and eosinophils, in the lungs of COVID- 19 patients. These findings are consistent with the results of our study. Their study showed that there was a moderate and insignificant increase in NKT cells in the lungs of COVID-19 patients. In addition, eosinophils also showed a non-significant increase [17]. However, the role of NKT cells in early eosinophilic infiltration has been previously reported in a mouse model of allergic asthma and neonatal RSV infection $[18,19]$. Moreover, $C D 3^{+} C D 16 / 56^{+}$ NKT cell levels were positively correlated positively with the percentage of eosinophils in BALF specimens from patients with eosinophilic pneumonia [20]. Although the association between elevated NKT cells and the pathogenesis of eosinophil-mediated inflammation is poorly understood, it may depend on the underlying airway epithelial injury caused by SARS-CoV-2 infection [21]. Subsequently, eosinophilic infiltration and degranulation appear to mediate lung inflammation and are associated with clinical manifestations [22]. Peripheral blood eosinophilia may initially present as eosinophil-mediated inflammation; however, it may also be absent or delayed [22], as consistently observed in patients diagnosed with COVID-19 (see complete blood count [CBC] results in Supplementary Fig. 3) [23].
As eosinophil-mediated inflammation in the lungs is associated with T-helper type 2 (Th2)-polarized immune responses [22], we next assessed viral antigen-specific antibody responses and focused on specific isotypes. We speculated that specific immune responses against the SARS-CoV-2 antigen are orchestrated by a Th2-biased immune environment, which can drive the preferential isotype switching of specific antibodies in B cells toward specific subclasses, such as immunoglobulin $A(\lg A)$ and $\lg E$ [24]. There was an increase in the levels of anti-N protein antibodies, including $\lg G 1, \lg G 3, \lg A$, and $\lg E$ isotypes $(\lg G 2$ and $\lg G 4$ were not detected) in the plasma from P1 and P2, who had severe pneumonia, whereas only moderate levels of $\lg G 1$ and $\lg G 3$ antibodies were observed in P3 with mild symptoms (Fig. 2C). Moreover, IgA and IgE antibodies were not detected at P3.

The administration of steroids in patients infected with other coronaviruses, such as Middle East respiratory syndrome (MERS) or SARS-CoV-1, resulted in delayed viral clearance with other short- and long-term side effects [25]. The World Health Organization (WHO), Centers for Disease Control and Prevention (CDC), and Infectious Disease Society of America (IDSA) guidelines do not recommend the treatment of COVID-19 patients with glucocorticoids, unless indicated otherwise, such as that during COPD exacerbation, until the Randomised Evaluation of COVID-19 Therapy trial (RECOVERY) trial was published [26]. However, systemic glucocorticoid therapy is generally considered the main treatment for acute eosinophilic pneumonia [22]. Consequently, we assumed that in patients with COVID-19, the systemic steroid (dexamethasone) that was used in the RECOVERY trial might have had a therapeutic effect on eosinophil-mediated lung inflammation via the above-mentioned mechanism. In our study, methylprednisolone was administered to patients with rapid and progressive pneumonia (P1 and P2); their oxygen demand decreased substantially and there was an improvement in their X-ray findings, although the blood glucose levels rose to 400 to $500 \mathrm{mg} / \mathrm{dL}$. This improvement was suspected to be the result of the steroid administration. Moreover, an analysis of 201 COVID-19 patients from Wuhan indicated a reduction in the mortality risk for patients with ARDS treated with methylprednisolone [27]. The ability of steroid treatment to decrease the mortality risk in patients with COVID-19 suggests that COVID-19 associated pneumonia is, in fact, an eosinophilic pulmonary inflammation. Therefore, future studies on possible thera- 
pies for eosinophilic pulmonary inflammation in COVID-19 patients need to be conducted, targeting eosinophilia and NKT cell response in the pulmonary tract [28-30]. Additionally, rapid and strong elevation of anti-viral antibodies, such as IgE, as we observed in a patient with severe COVID-19, must be taken into consideration for plasma therapy (i.e., potential risk of type I hypersensitivity) [31].

This study had several limitations. As the study data consisted of a case series of only three patients and the data was collected retrospectively, other important clinical variables that contribute to the differential diagnosis of other obvious causes of eosinophilia, such as thyroid disease, parasite infection, malignancy, and allergic disease, may have been missed.

Notwithstanding these limitations, the results of our study showed that COVID-19 can lead to eosinophil-mediated inflammation via systemic elevation of NKT cells. The infiltration of NKT cells into infected lungs may be associated with COVID-19 pneumonic inflammation.

\section{KEY MESSAGE}

1. We report an association between eosinophilic pulmonary inflammation and elevated natural killer $T$ (NKT) cell responses in three patients with coronavirus disease 2019 (COVID-19). Systemic increase in NKT cells and their infiltration into infected lungs may be associated with eosinophilic lung inflammation in patients with COVID-19.

2. The pathogenesis of severe COVID-19 pneumonia remains unknown. We describe the role of NKT cells and eosinophils in the pathogenesis of severe COVID-19 pneumonia. Our study results provide preliminary evidence in support of steroid administration in patients with severe COVID-19 pneumonia, although further follow-up studies are needed.

\section{Conflict of interest}

No potential conflict of interest relevant to this article was reported.

\section{Acknowledgments}

This study was supported by a research fund from the Chosun University Hospital, 2020. Yuri Kim, Uni Park, Na-
Young $\mathrm{Ha}$, and Hyoree Park received scholarships from the BK21-plus education program funded by the National Research Foundation of Korea.

We are grateful to all nurses (Myung Sook Kim, Jin Yeong Park, A Ra Kim, Hye Yeon Lee, Hye Mi Park, and So Sun Jeon from the Department of Infection Control; and Sun Ok Joo, Seong Hee Kang, Hye Jin Kim, Yun Hee Choi, Ji Hye son, Jeong A Hwang, Sun a Jeong, Da Jeong Mun, Seung Hyun Lee, Seo Hee Seo, Yeong Ji Lim, Geum Bit Hwang, Eun Jeong Gong, Han Sol Hong, Ji Seon Jeong, Su Yeon Go, Na Ri Lee, Ja In Moon, Min Seo Kim, Hyun Young Lee, Sang Young Kim, Bo Ra Lim, Song Won Lee, Ju Jin Kim, Ha Neul Seon, Yea Ji Moon, Jin Young Jo, and Da Min Park from a nationally designated quarantine ward at Chosun University Hospital) and patients involved in the study.

\section{REFERENCES}

1. Zhu $N$, Zhang $D$, Wang $W$, et al. A novel coronavirus from patients with pneumonia in China, 2019. N Engl J Med 2020;382:727-733.

2. Coronaviridae Study Group of the International Committee on Taxonomy of Viruses. The species severe acute respiratory syndrome-related coronavirus: classifying 2019-nCoV and naming it SARS-CoV-2. Nat Microbiol 2020;5:536-544.

3. Kim ES, Chin BS, Kang CK, et al. Clinical course and outcomes of patients with severe acute respiratory syndrome coronavirus 2 infection: a preliminary report of the first 28 patients from the Korean cohort study on COVID-19. J Korean Med Sci 2020;35:e142.

4. Zhou F, Yu T, Du R, et al. Clinical course and risk factors for mortality of adult inpatients with COVID-19 in Wuhan, China: a retrospective cohort study. Lancet 2020;395:1054-1062.

5. Conway SP, Pond MN, Bowler I, et al. The chest radiograph in cystic fibrosis: a new scoring system compared with the Chrispin-Norman and Brasfield scores. Thorax 1994;49:860862.

6. Barnig C, Alsaleh $\mathrm{G}$, Jung N, et al. Circulating human eosinophils share a similar transcriptional profile in asthma and other hypereosinophilic disorders. PLoS One 2015;10:e0141740.

7. Das R, Sant'Angelo DB, Nichols KE. Transcriptional control of invariant NKT cell development. Immunol Rev 2010;238:195215.

8. Yu YR, Hotten DF, Malakhau Y, et al. Flow cytometric analysis of myeloid cells in human blood, bronchoalveolar lavage, and 
lung tissues. Am J Respir Cell Mol Biol 2016;54:13-24.

9. Godfrey DI, MacDonald HR, Kronenberg M, Smyth MJ, Van Kaer L. NKT cells: what's in a name? Nat Rev Immunol 2004:4:231-237.

10. Dentone $C$, Vena A, Loconte $M$, et al. Bronchoalveolar lavage fluid characteristics and outcomes of invasively mechanically ventilated patients with COVID-19 pneumonia in Genoa, Italy. BMC Infect Dis 2021;21:353.

11. Liao M, Liu Y, Yuan J, et al. Single-cell landscape of bronchoalveolar immune cells in patients with COVID-19. Nat Med 2020;26:842-844.

12. Pandolfi L, Fossali T, Frangipane $V$, et al. Broncho-alveolar inflammation in COVID-19 patients: a correlation with clinical outcome. BMC Pulm Med 2020;20:301.

13. Fraenkel DJ, Bardin PG, Sanderson G, Lampe F, Johnston SL, Holgate ST. Lower airways inflammation during rhinovirus colds in normal and in asthmatic subjects. Am J Respir Crit Care Med 1995;151(3 Pt 1):879-886.

14. Harrison AM, Bonville CA, Rosenberg HF, Domachowske JB. Respiratory syncytical virus-induced chemokine expression in the lower airways: eosinophil recruitment and degranulation. Am J Respir Crit Care Med 1999;159:1918-1924.

15. Jeon EJ, Kim KH, Min KH. Acute eosinophilic pneumonia associated with 2009 influenza A (H1N1). Thorax 2010;65:268270.

16. LeMessurier KS, Rooney R, Ghoneim HE, et al. Influenza A virus directly modulates mouse eosinophil responses. J Leukoc Biol 2020;108:151-168.

17. Cavalli E, Petralia MC, Basile MS, et al. Transcriptomic analysis of COVID-19 lungs and bronchoalveolar lavage fluid samples reveals predominant $B$ cell activation responses to infection. Int J Mol Med 2020;46:1266-1273.

18. Bilenki L, Yang J, Fan Y, Wang S, Yang X. Natural killer T cells contribute to airway eosinophilic inflammation induced by ragweed through enhanced IL-4 and eotaxin production. Eur J Immunol 2004;34:345-354.

19. Lee SY, Noh Y, Goo JH, et al. Natural killer T cell sensitization during neonatal respiratory syncytial virus infection induces eosinophilic lung disease in re-infected adult mice. PLoS One 2017;12:e0176940.

20. Papakosta D, Manika K, Kyriazis G, et al. Bronchoalveolar lavage fluid eosinophils are correlated to natural killer cells in eosinophilic pneumonias. Respiration 2009;78:177-184.

21. Kimura $H$, Francisco D, Conway $M$, et al. Type 2 inflammation modulates ACE2 and TMPRSS2 in airway epithelial cells. J Allergy Clin Immunol 2020;146:80-88.

22. De Giacomi F, Vassallo R, Yi ES, Ryu JH. Acute eosinophilic pneumonia: causes, diagnosis, and management. Am J Respir Crit Care Med 2018;197:728-736.

23. Qin C, Zhou L, Hu Z, et al. Dysregulation of immune response in patients with coronavirus 2019 (COVID-19) in Wuhan, China. Clin Infect Dis 2020;71:762-768.

24. Scott-Taylor TH, Axinia SC, Amin S, Pettengell R. Immunoglobulin G; structure and functional implications of different subclass modifications in initiation and resolution of allergy. Immun Inflamm Dis 2018;6:13-33.

25. Russell CD, Millar JE, Baillie JK. Clinical evidence does not support corticosteroid treatment for 2019-nCoV lung injury. Lancet 2020;395:473-475.

26. RECOVERY Collaborative Group, Horby P, Lim WS, et al. Dexamethasone in hospitalized patients with COVID-19. N Engl J Med 2021;384:693-704.

27. Wu C, Chen X, Cai Y, et al. Risk factors associated with acute respiratory distress syndrome and death in patients with coronavirus disease 2019 pneumonia in Wuhan, China. JAMA Intern Med 2020;180:934-943.

28. Jacobson KA, Tosh DK, Jain S, Gao ZG. Historical and current adenosine receptor agonists in preclinical and clinical development. Front Cell Neurosci 2019;13:124.

29. Heulens $N$, Korf $H$, Janssens $W$. Innate immune modulation in chronic obstructive pulmonary disease: moving closer toward vitamin D therapy. J Pharmacol Exp Ther 2015;353:360-368.

30. Panarese A, Shahini E. Letter: COVID-19, and vitamin D. Aliment Pharmacol Ther 2020;51:993-995.

31. Chen L, Xiong J, Bao L, Shi Y. Convalescent plasma as a potential therapy for COVID-19. Lancet Infect Dis 2020;20:398400. 


\section{SUPPLEMENTARY METHODS}

\section{Cytological analysis}

In P1 and P2, bronchoscopy was performed under conscious sedation using intravenous midazolam. Heart rate, respiratory rate, blood pressure, and oxygen saturation were monitored before, during, and after the procedure. Bronchoalveolar lavage fluid (BALF) was performed with a single-use disposable bronchoscope (Ambuß aScope ${ }^{\mathrm{TM}} 4$, Ambu A/S, Ballerup, Denmark) in a wedged position within a subsegmental bronchus by a pulmonologist. Sterile $0.9 \% \mathrm{NaCl}$ saline was instilled in the selected subsegmental bronchus in sequential aliquots $(30,30$, and $40 \mathrm{~mL})$. After saline instillation, the fluid was immediately recovered into the same syringe by gentle and continuous manual aspiration. BAL and sputum cytology samples were obtained and smeared onto glass slides immediately after collection. The slides were then immediately fixed with 95\% ethanol in a Coplin jar. After fixing, hematoxylin and eosin (H\&E) staining was performed. Concentration of eosinophil cationic protein in respiratory specimens was measured using clinical diagnosis service from Seoul clinical laboratory (Seoul, Korea) after gamma-irradiation (30 kGy, Soyagreentech, Seoul, Korea).

\section{Flow cytometry}

Peripheral blood mononuclear cells (PBMCs) were prepared by standard density gradient centrifugation using Histopaque-1077 and 1119 (Sigma-Aldrich, St. Louis, MO, USA) in a Biosafety Level 3 laboratory at Seoul National University. Blood leukocytes and PBMCs were cryopreserved in liquid nitrogen or directly stained with antibodies listed below for flow cytometry in the same laboratory. Dead cells were stained with Zombie Aqua Fixable Viability Dye (BioLegend, San Diego, CA, USA). Cells were stained with the following sets of antibodies differentially labeled with indicated fluorochrome; anti-CD4-Alexa488, anti-CD8-PerCP or APC, anti-CD14-BV605, anti-CD16-Alexa700, anti-CD24-BV421, anti-CD45-PerCP-Cy5.5, anti-CD206-Alexa488, anti-HLA-DR-BV711 (from BioLegend), anti-CD3-PE-CF594 or Pacific blue, anti-CD20-APC/ $\mathrm{H7}$, and anti-CD56-APC (from BD Bioscience, Franklin
Lakes, NJ, USA). PE-conjugated CD1d/PBS57 tetramers obtained from the National Institute of Health Tetramer Core Facility (Bethesda, MD, USA) were kindly supplied by Dr. Doo Hyun Cheong at Seoul National University College of Medicine. Cell were then fixed with a fixation buffer (BD Bioscience) and analyzed using a FACS Fortessa II flow cytometer (BD Biosciences). Data were analyzed using Flowjo software (Tree Star, Ashland, OR, USA).

\section{Enzyme-linked immunosorbent assay}

To assess SARS-CoV N protein-specific antibody responses, 96-well immunoassay plates (Nunc, Waltham, MA, USA) were coated with $100 \mu \mathrm{L}$ of purified antigen at a concentration of $1 \mu \mathrm{g} / \mathrm{mL}$ at $4^{\circ} \mathrm{C}$ for overnight. The plates were then blocked for 2 hours at room temperature (RT) with PBS containing 5\% skim milk. One hundred microliters of serially diluted plasma samples were incubated for 2 hours at RT. After washing with PBS containing $0.05 \%$ Tween 20 (0.05\% PBST), horseradish peroxidase-conjugated mouse anti-human immunoglobulin $\mathrm{G} 1$ ( $\lg \mathrm{G} 1), \lg \mathrm{g} 2, \lg G 3, \lg \mathrm{G} 4$, $\lg \mathrm{A}$, or $\lg \mathrm{E}$ antibody (Southern Biotech, Birmingham, AL, USA) was added and incubated for 1 hour at RT. Wells were then washed with $0.05 \%$ PBST and incubated with a 3,3',5,5'-tetramethylbenzidine peroxidase substrate solution (KPL, Gaithersburg, MD, USA) for 10 minutes. The reactions were stopped by adding $1 \mathrm{M}$ phosphoric acid solution. Absorbance was measured at $450 \mathrm{~nm}$ using a microplate reader (Beckman Coulter, Brea, CA, USA). The cut-off titer for the enzyme-linked immunosorbent assay (ELISA) was determined as the lowest titer showing an optical density (OD) over the mean OD plus $3 \times$ standard deviation from three control plasma samples (diluted 1:10).

\section{Statistical analysis}

Data was analyzed using the Graph Pad Prism 5.01 software (GraphPad Software, La Jolla, CA, USA). Statistical analysis was performed using one-way analysis of variance (ANOVA) followed by Newman-Keuls $t$ test for comparisons of values among different groups. A $p<0.05$ was considered statistically significant. 

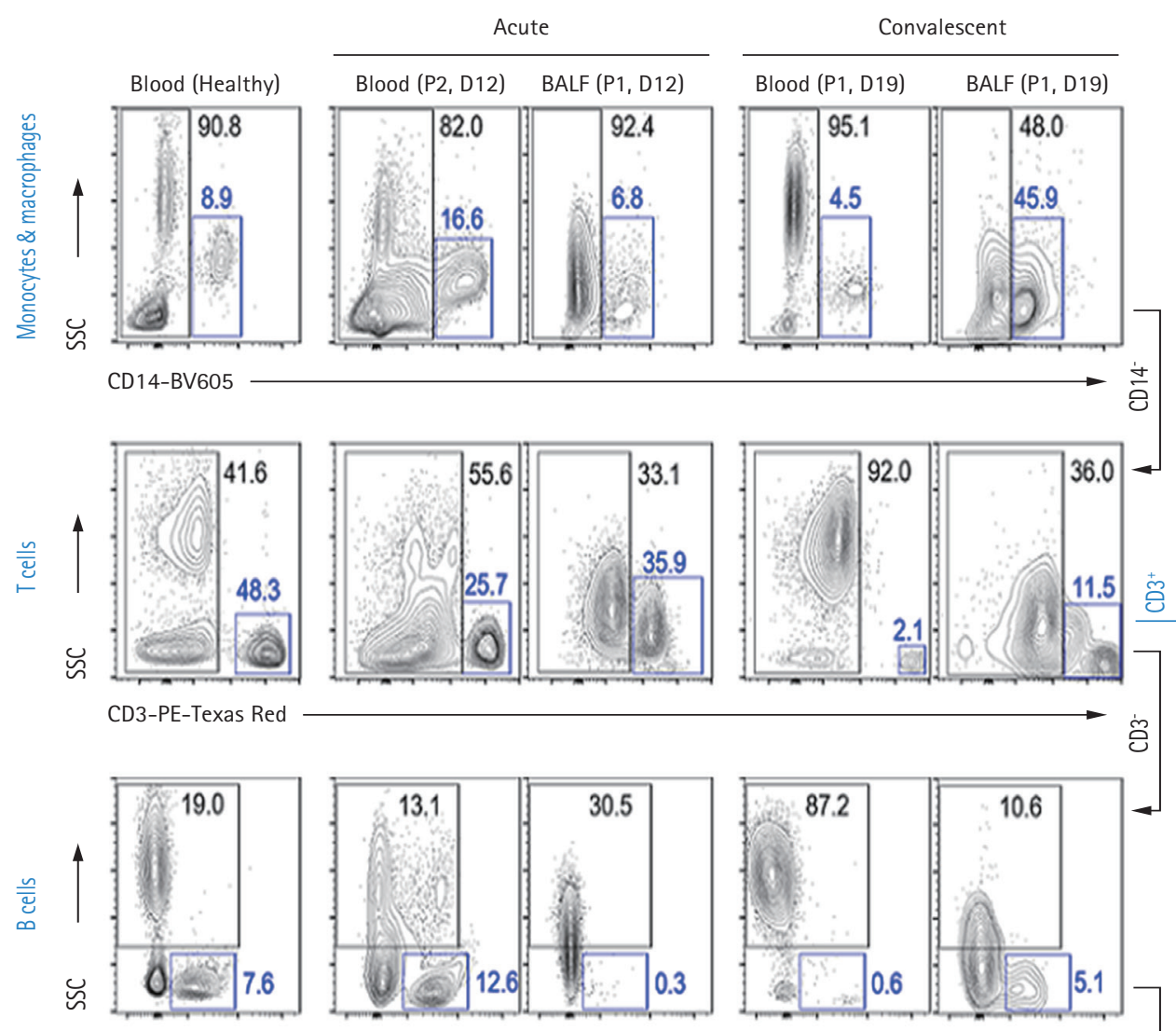

CD20-APC-Cy7
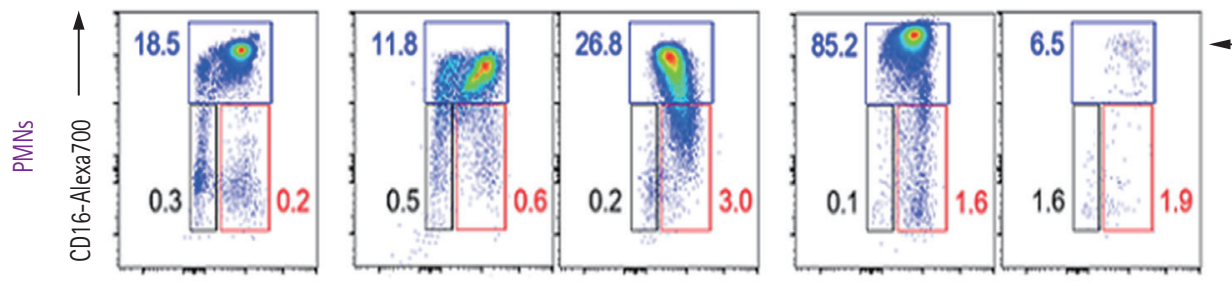

CD24-BV421
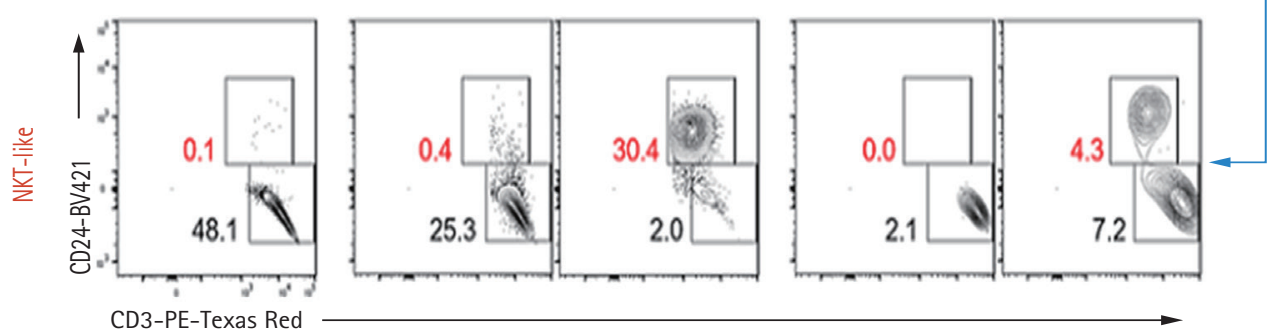

Supplementary Figure 1. Relative frequencies of leukocyte subtypes in blood and bronchoalveolar lavage fluid (BALF) specimens from patients with severe coronavirus disease 2019 (COVID-19). Flow cytometric analyses of leukocytes in blood and BALF specimens collected from two patients with severe COVID-19 (P1 and P2) during acute and convalescent phases were performed after staining with indicated antibodies. The patients' ID and sample collection day (days after symptom onset) are indicated at the top. Blood leukocytes collected from a healthy volunteer were used as a control for flow cytometry (left panels). The relative frequencies of specific leukocytes subtype population are indicated in each window. In polymorphonuclear (PMN) population, the relative frequencies of neutrophils (blue), eosinophils (red), and mast cells (black) are color-gated based on the expression levels of CD16 and CD24. All frequencies are relative levels of CD45 leukocytes. SSC, side scatter; PE, phycoerythrin; APC, allophycocyanin; NKT, natural killer T. 


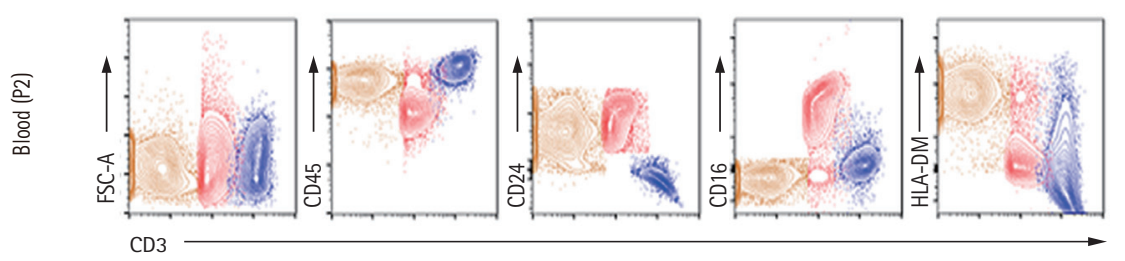

\section{A}
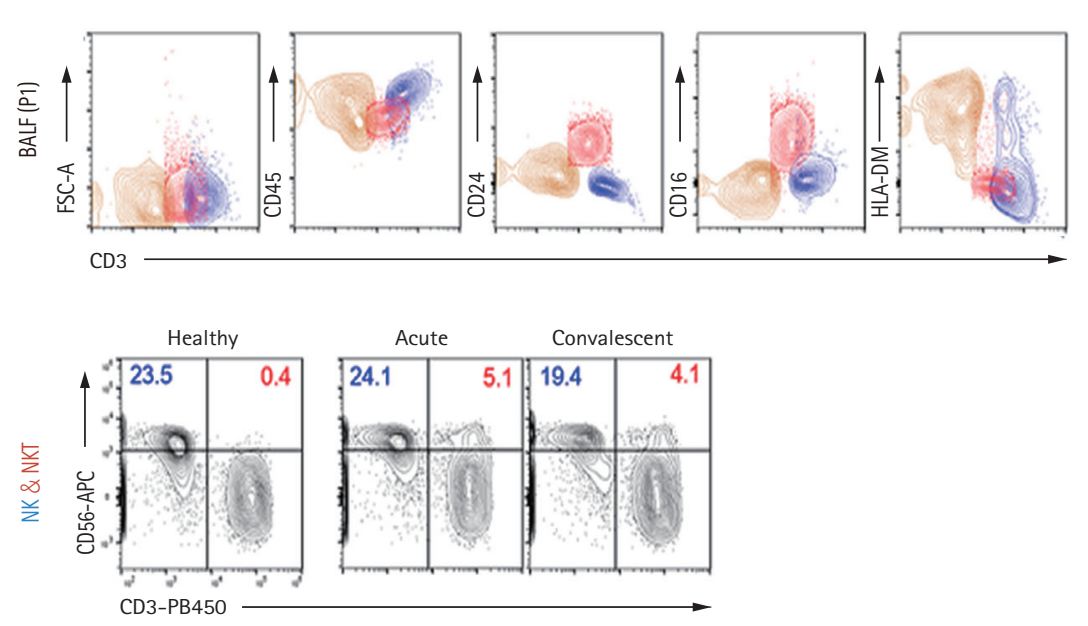

B

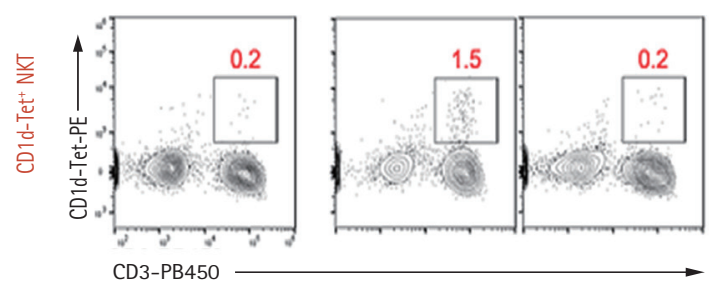

\section{C}
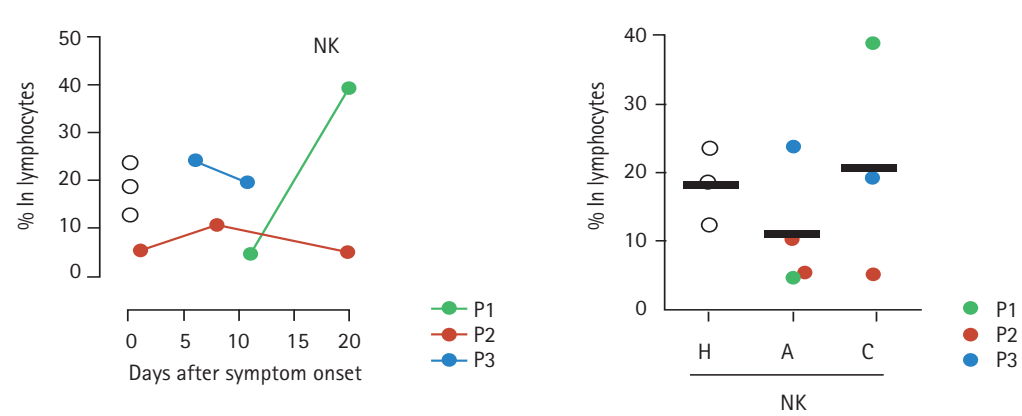

Supplementary Figure 2. Characterization of natural killer (NK) and natural killer T (NKT) cells in blood and bronchoalveolar lavage fluids (BALFs) and peripheral blood mononuclear cells (PBMCs) from patients with coronavirus disease 2019 (COVID-19). (A) Relative levels of leukocyte makers of B (brown), NKT-like (red), and T cells (blue) in blood and BALF specimens from patients with severe COVID-19. These cell types are gated population from Supplementary Fig. 1. (B) Representative contour plots identifying NK, NKT, and CD1d-tetramer (Tet)-positive NKT (invariant NKT) cells using PBMCs from the patients with COVID-19. (C) Kinetic changes in the relative frequencies of CD3-/CD56+ NK cells among lymphocytes from the indicated patients are presented (right panel). The relative frequencies of each immune cell type from three healthy volunteers were included as controls (open circles). The relative frequencies of CD3-/CD56+ NK cells among lymphocytes from the patients were rearranged based on the collection periods, i.e., acute phase $(A)$ and convalescent phase $(C)$. The relative frequencies of NK cells from three healthy volunteers were included as controls ( $\mathrm{H}$, open circles). Black bars indicate mean values. FSC-A, forward scatter-A; HLA-DM, human leukocyte antigen DM; APC, allophycocyanin; PE, phycoerythrin. 

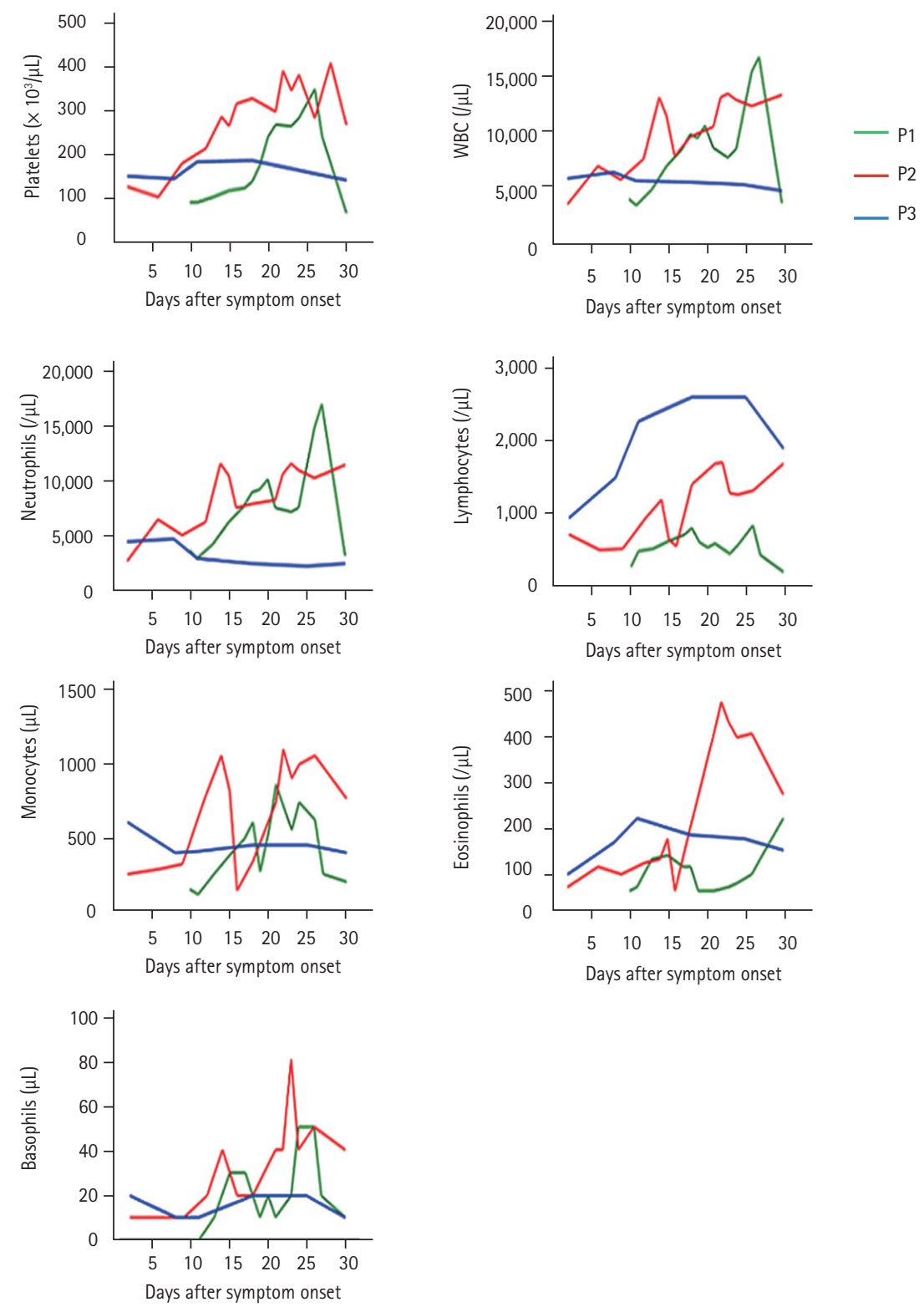

Supplementary Figure 3. Kinetics of complete blood counts for the three patients with coronavirus disease 2019 (COVID-19). WBC, white blood cell. 